\title{
Low-Cost and Long-Range Node-Assisted WiFi Backscatter Communication for 5G-Enabled IoT Networks
}

\author{
Zhimin Wang $\mathbb{D}^{1},{ }^{1}$ Li Feng $\mathbb{D}^{1},{ }^{1}$ Shumin Yao, ${ }^{1}$ Kan Xie, ${ }^{2,3}$ and Yuqiang Chen ${ }^{4}$ \\ ${ }^{1}$ Faculty of Information Technology, Macau University of Science and Technology, Taipa, Macau 999078, China \\ ${ }^{2}$ Information Processing and System Integration of IoT, Ministry of Education of the P.R.C., Guangzhou 510006, China \\ ${ }^{3}$ Guangdong-HongKong-Macao Joint Laboratory for Smart Discrete Manufacturing, Guangzhou 510006, China \\ ${ }^{4}$ School of Information and Communication Engineering, Guangzhou Maritime University, Guangzhou 510725, China
}

Correspondence should be addressed to Li Feng; lfeng@must.edu.mo

Received 9 May 2021; Revised 12 June 2021; Accepted 7 July 2021; Published 21 July 2021

Academic Editor: Xingwang Li

Copyright (C) 2021 Zhimin Wang et al. This is an open access article distributed under the Creative Commons Attribution License, which permits unrestricted use, distribution, and reproduction in any medium, provided the original work is properly cited.

\begin{abstract}
The fifth-generation-enabled Internet of Things (5G-enabled IoT) has been considered as a key enabler for the automation of almost all industries. In 5G-enabled IoT, resource-limited passive devices are expected to join the IoT using the WiFi backscatter communication (WiFi-BSC) technology. However, WiFi-BSC deployment is currently limited due to high equipment cost and short transmission range. To address these two drawbacks, in this paper, we propose a low-cost and longrange node-assisted WiFi backscatter communication scheme. In our scheme, a WiFi node can receive backscatter signals using two cheap regular half-duplex antennas (instead of using expensive full-duplex technique or collaborating with multiple other nodes), thereby reducing the equipment cost. Besides, WiFi nodes can help relay backscatter signals to remote 5G infrastructure, greatly extending the backscatter's transmission range. We then develop a theoretical model to analyze the throughput of WiFiBSC. Extensive simulations verify the effectiveness of our scheme and the accuracy of our model.
\end{abstract}

\section{Introduction}

The fifth-generation-enabled Internet of Things (5G-enabled IoT) is considered to be a key enabler for automation of almost all industries [1-5]. In a typical 5G-enabled IoT, a $5 \mathrm{G}$ customer premise equipment (5G-CPE) is introduced to bridge a WiFi-based IoT network and a 5G base station [6], as shown in Figure 1. In this solution, the 5G-CPE may first use its WiFi module to collect data from the WiFi-based IoT devices and then upload these data to a remote cloud server timely using its $5 \mathrm{G}$ module. Hence, this solution can take full advantage of 5G's high bandwidth and low latency, as well as WiFi's ubiquity.

Besides WiFi, backscatter communication (BSC) is another popular wireless technology in IoT [7]. BSC is designed for communications between tags (which are severely resource-limited and battery-free) and readers. With BSC, tags first harvest energy from ambient signals, then convey information by modulating and reflecting ambient sig- nals (instead of generating signals by themselves), while readers are dedicatedly designed to receive the reflected signals from tags. As a recent advance, WiFi-based BSC (WiFi-BSC) [8-11] has been proposed. With WiFi-BSC, tags may harvest energy and convey information by utilizing ubiquitous WiFi signals, while $\mathrm{WiFi}$ nodes with selfinterference cancellation capability (which is called WiFi reader) may receive the reflected signals from tags. WiFiBSC opens up a promising opportunity for tags to join WiFi-based IoT networks, but WiFi readers are expensive because they are required to support costly full-duplex techniques or collaborate with other WiFi nodes to implement the self-interference cancellation. On the other hand, the transmission range of WiFi-BSC is very short (say, $1 \mathrm{~m}$ to $54 \mathrm{~m}[9,10])$. As a result, current WiFi-BSC deployment is very limited.

1.1. Motivation. In recent years, the multiuser, multi-input, and multioutput (MU-MIMO) technology has been intro- 


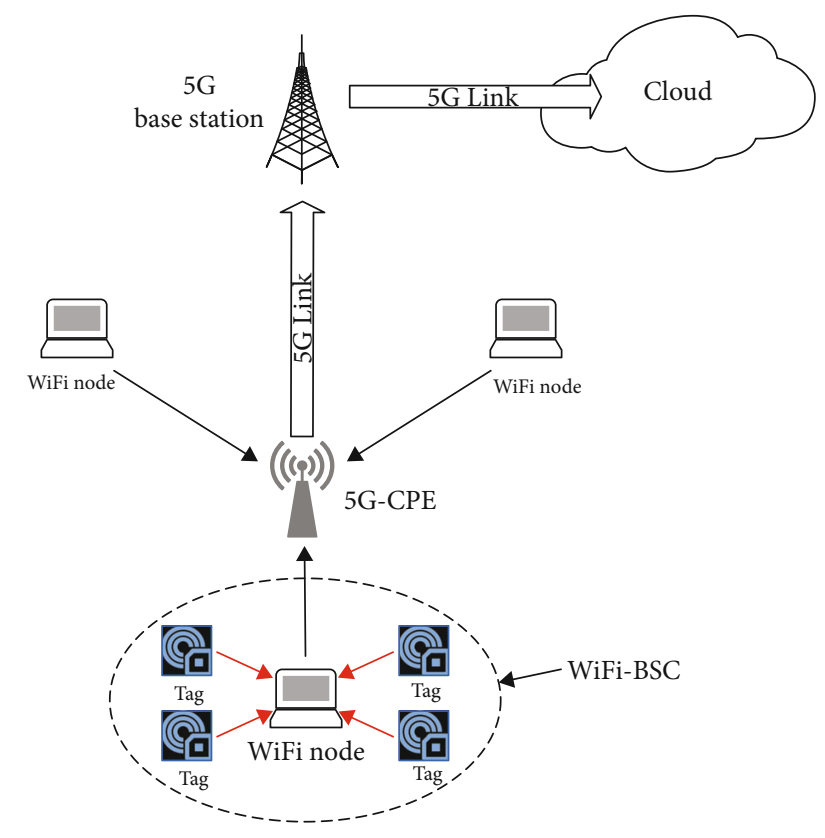

Figure 1: A 5G-enabled IoT network.

duced to WiFi. With MU-MIMO, a WiFi node can use regular half-duplex antennas to receive different signals from multiple nodes simultaneously and then distinguish these signals for further processing. This feature motivates us to apply MU-MIMO in WiFi-BSC for enabling conventional WiFi nodes to act as WiFi readers, without utilizing costly full-duplex technique and collaborating with other nodes.

In addition, the transmission range of $\mathrm{WiFi}$ (up to $1000 \mathrm{~m}[12,13])$ is much longer than that of WiFi-BSC. This motivates us to let WiFi nodes relay tags' information toward $5 \mathrm{G}-\mathrm{CPE}$, greatly extending the transmission range of the WiFi-BSC.

1.2. Contribution. In this paper, we propose a low-cost and long-range node-assisted WiFi-BSC scheme. Our contributions can be summarized as follows.

(1) Propose a low-cost WiFi-BSC design. Our design only requires a conventional WiFi node to be equipped with two regular half-duplex antennas, so as to adopt MU-MIMO to decode the reflected signals from tags. This avoids adopting costly fullduplex technique and collaborating with other nodes for extracting reflected signals of tags, thereby saving equipment cost greatly

(2) Propose a long-range node-assisted WiFi-BSC design. Our design lets WiFi nodes relay tags' information to a remote 5G-CPE, hence extending the transmission range of the tags significantly

(3) Develop a theoretical model to analyze the throughput of our scheme, and verify the accuracy of our model via extensive simulations. The results show that our design significantly outperforms existing WiFi-BSC schemes in terms of throughput
1.3. Related Works. In recent years, BSC has quickly become a key technology for low-power wireless communication systems [14] and therefore has received lots of attention in the research community. Below, we present related works of BSC in terms of physical (PHY) layer and medium access control (MAC) layer schemes.

1.3.1. PHY Layer Schemes. In the PHY layer, many works are dedicated to achieving feasible BSC. For example, Liu et al. proposed ambient backscatter that enables two tags to communicate with each other by reflecting ambient TV signals [7]. However, in practical IoT systems, tags are more commonly covered by WiFi signals instead of TV signals. To this end, WiFi backscatter [10] enables tags to reflect ambient WiFi signals in their transmissions and uses an off-the-shelf WiFi device to receive and decode reflected signals. However, the decoding accuracy of this approach is low, because the WiFi node receives both the reflected signal and the ambient signal and has a difficulty in separating these two signals. To improve the decoding accuracy, BackFi [8] is proposed to enable the reader to have prior knowledge of the ambient signal. In this approach, a WiFi node equipped with a fullduplex transceiver is introduced to perform regular WiFi transmission and backscatter reception simultaneously. When decoding the reflected signals, the node adopts the self-interference cancellation (SIC) technique to cancel the ambient signals sent by itself. Nevertheless, deploying a full-duplex transceiver is costly. To avoid deploying a fullduplex transceiver, HitchHike [9] lets tags reflect ambient WiFi signals from a WiFi band to a dedicated band. At the receiving end, there are two WiFi nodes connecting to the same backend server. One node receives the reflected signal over the dedicated band, while the other node receives the regular signal over the WiFi band. Then, the server collects these two signals and extracts tags' data from the reflected signal by comparing these two signals. This design requires collaborations between multiple WiFi nodes, which is still expensive. Different from the above approaches, without deploying a full-duplex transceiver and assigning a dedicated band for BSC in our design, we enable a half-duplex WiFi node to receive and decode the backscatter signals using only two regular antennas.

Besides, some works focus on improving the PHY layer security (PLS) of BSC. For example, in [15], Li et al. investigated the impact of in-phase and quadrature-phase imbalance on the security performance of BSC systems and found out that there exists a trade-off between reliability and security. Further, they proposed a system parameter adjustment scheme to prevent BSC from being eavesdropped. Reference [16] investigated the joint effects of residual hardware impairments, channel estimation errors, and imperfect successive interference cancellation on the PLS of BSC and proposed an artificial noise scheme to prevent BSC form being eavesdropped. Reference [17] studied the PLS of BSC in the case where cognitive radio is introduced in conjunction with BSC. The authors also optimized the performance trade-off between reliability and security of BSC by adjusting system parameters. It is worth pointing out that the PLS of BSC is out of scope of this paper. 
1.3.2. MAC Layer Schemes. Along with PHY layer achievements, some works focus on proposing efficient medium access control (MAC) protocol for BSC. For example, [18] proposed a distributed MAC protocol for tag-to-tag communications. When ambient signals exist, tags contend for transmissions, and the loser tags harvest energy while the winner tag performs transmissions. There are also MAC designs for tag-to-WiFi communications. Most of them assumed a full-duplex WiFi access point (AP) that can emit ambient signals and receive the reflected signals from tags simultaneously. For example, Ma et al. proposed a demandbased MAC scheme for WiFi backscatter communication [11]. In this scheme, when having a demand for collecting data from tags, the full-duplex WiFi AP ceases all WiFi transmissions and sends a period of ambient signals, and tags contend and transmit data to the AP during this period. Reference [19] proposed a rendezvous scheme for sporadic BSC. In this scheme, a full-duplex WiFi AP divides the time into frames with equal length; each frame is further divided into subperiods for coordination, WiFi transmission, and BSC, respectively. Tags perform contentions in the coordination subperiods and perform transmissions (to the AP) in the BSC subperiods. Then, this idea is extended by [20], where the full-duplex WiFi AP adopts the deep reinforcement learning technique to learn the optimal time-division strategy. Different from the above approaches, we focus on extending the range of BSC, with the assistance of regular half-duplex WiFi nodes.

The rest of this paper is organized as follows. Section 2 details our design. In Section 3, we theoretically analyze the performance of our design. Section 4 validates the accuracy of our theoretical model. Section 5 concludes this paper.

\section{Our Design}

In this section, we detail our low-cost and long-range nodeassisted WiFi backscatter communication scheme for 5Genabled IoT. The scheme consists of a physical (PHY) layer design and a MAC layer design. Below, we first present an overview of the scheme and then detail two designs.

2.1. Design Overview. In our design, we consider a 5Genabled IoT network, which consists of one 5G-CPE, multiple WiFi nodes, and multiple backscatter tags. Adopting off-the-shelf hardware only, we propose a design to achieve an objective as follows. Backscatter tags first transmit data to their nearby WiFi nodes; then, the WiFi node forwards the backscatter data to the remote 5G-CPE.

In the PHY layer, whenever a WiFi signal is sent, the tag uses the signal as an excitation signal to perform a tag-to-tag communication or a tag-to-WiFi communication. During the tag-to-WiFi communication, a WiFi node can extract backscatter data from ambient WiFi signals using only two regular half-duplex antennas, instead of being equipped with expensive full-duplex receivers or collaborating with multiple nodes.

In the MAC layer, we divide the IoT network into multiple subcells (as shown in Figure 2), where each subcell consists of a WiFi node and multiple tags. We propose a MAC

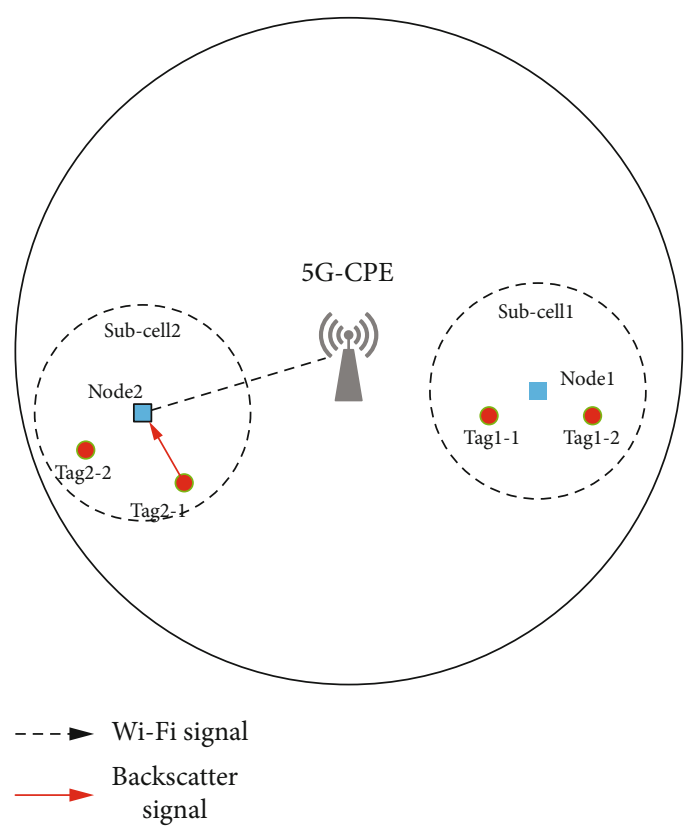

Figure 2: Design overview: Tag2-1 first transmits its data to Node2, and then Node2 forwards the backscatter data to the remote 5GCPE.

layer protocol as follows. First, WiFi nodes follow the WiFi standard to contend for the channel. Then, the WiFi winner node starts a WiFi transmission, while the tags in its subcell harvest energy from the transmission signal. At the same time, other tags adopt our proposed scheme to contend and transmit data to the WiFi nodes in their subcells.

Below, we detail our PHY layer design and our MAC layer design in sequence.

2.2. PHY Layer Design. In our PHY layer design, we assume that each WiFi node has two antennas: antenna 1 (AT1) and antenna 2 (AT2). When a WiFi node transmits signals, it only activates its AT1. When a WiFi node receives signals, it activates both AT1 and AT2.

As shown in Figure 3, when a node (say, Node1) sends a WiFi signal to 5G-CPE, other nodes (say, Node2) and the tags (say, Tag2-1 and Tag2-2) can sense this signal. Using the WiFi signal as an excitation signal, a tag (say, Tag2-1) can adopt the Manchester technology to send information to other tags (say, Tag2-2). It can also adopt the $M$-ary phase shift keying (M-PSK) technology to perform a high-rate transmission to a WiFi node (say, Node2). Below, we detail the tag-to-tag communication and the tag-to-WiFi communication.

2.2.1. Tag-to-Tag Communication. We adopt the Manchester technology in tag-to-tag communication. Below, we introduce the Manchester modulation and demodulation under the ambient WiFi signals, respectively.

(1) Modulation. When performing the Manchester modulation, a tag changes the amplitude of its received WiFi signal 


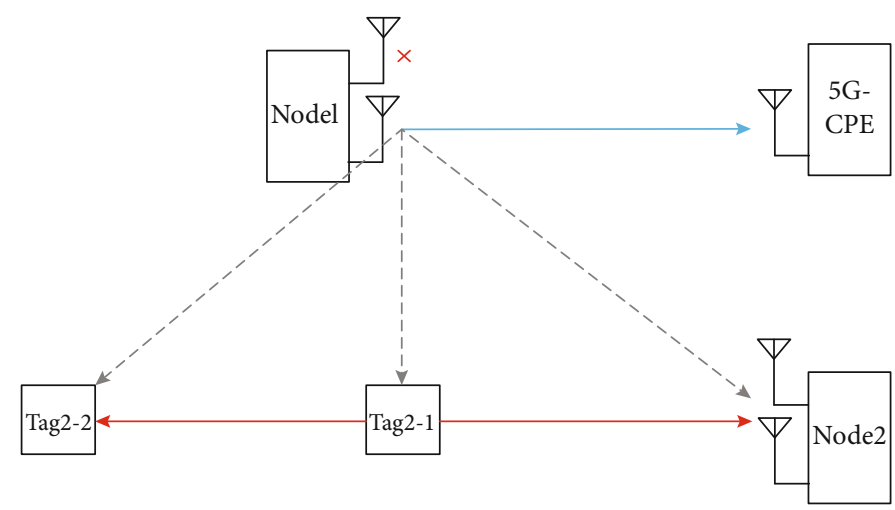

FIgURE 3: PHY overview: Nodel transmits its data to the 5G-CPE (in blue), and the transited signal (in grey) is also received by Tag2-1, Tag22, and Node2. Tag2-1 uses this WiFi signal as excitation signal and transmits its data to Tag2-2 and Node2 (in red).

$z(t)$ and then reflects the signal. Therefore, the backscatter signal $x_{b}(t)$ reflected by the tag can be expressed as follows:

$$
x_{b}(t)=m(t) \cdot z(t), 0 \leq t<T \text {, }
$$

where

$$
m(t)= \begin{cases}s(t), & \text { bit }=0 \\ -s(t), & \text { bit }=1\end{cases}
$$

is the backscatter baseband signal and

$$
s(t)= \begin{cases}1, & 0 \leq t<\frac{T}{2}, \\ 0, & \frac{T}{2} \leq t<T .\end{cases}
$$

(2) Demodulation. Each tag uses energy detection technique to demodulate a backscatter signal $y_{b}(t)=h_{b b}(t) * x_{b}(t)$ (ignoring noise) received from other tags, where $h_{b b}(t)$ is the impulse response of the channel between tags. We define

$$
\begin{gathered}
P_{1}=\int_{0}^{\frac{T}{2}}\left(y_{b}(t)\right)^{2} d t, \\
P_{2}=\int_{T / 2}^{T}\left(y_{b}(t)\right)^{2} d t .
\end{gathered}
$$

When $P_{1} \geq P_{2}$, the tag regards that $y_{b}(t)$ carries a bit " 0 "; otherwise, it regards that $y_{b}(t)$ carries a bit " 1 ."

2.2.2. Tag-to-WiFi Communication. We adopt the M-PSK technology in tag-to-WiFi communication. Below, we explain M-PSK modulation on tags and the demodulation on nodes sequentially.

(1) Modulation. In the M-PSK scheme, bits are represented by phases of symbols. We take the 4-PSK scheme (i.e., $M=$ 4) as an example to explain the modulation process. As shown in Figure 4, 4-PSK has 4 valid constellation points e $=\left\{e^{j \theta_{1}}, e^{j \theta_{2}}, e^{j \theta_{3}}, e^{j \theta_{4}}\right\}$; these constellation points correspond to bit pairs $\mathbf{B}=\{11,01,00,10\}$. When performing modulation, $m(t)$ in (1) is set according to the mapping relationship between e and B. For example, because " 10 " corresponds to $e^{j \theta_{4}}$, when modulating “ 10 ," we set $m(t)=e^{j \theta_{4}}$.

(2) Demodulation. In the case shown in Figure 2, Node2 receives regular WiFi signals $x_{w}(t)$ from Nodel and reflected signals $x_{b}(t)$ from Tag2-1 simultaneously. Because Node2 activates its AT1 and AT2 during its receptions, a MUMIMO system is formed, as shown in Figure 5. In this system, Node1's AT1 and the Tag2-1 are the inputs, while Node2's AT1 and AT2 are outputs. Benefitting from this system, Node 2 can easily extract $m(t)$ from $x_{b}(t)$ and then can decode backscatter data from $m(t)$ as follows.

Step 1. Extracting the backscatter baseband signal.

Because Node 2 activates two antennas in its reception, we can use a vector $\mathbf{y}$ to represent Node2's received signal:

$$
\mathbf{y}=\left[\begin{array}{l}
y_{1}(t) \\
y_{2}(t)
\end{array}\right],
$$

where $y_{i}(t)$ is the signal received by AT $i$ and $i=1,2$.

Taking into account the signal fading of $x_{w}(t)$ and $x_{b}(t)$ in the MU-MIMO system, (5) can be expanded as follows:

$$
\begin{aligned}
\text { (1) } \mathbf{y}= & {\left[\begin{array}{l}
x_{w}(t) * h_{11}(t)+x_{b}(t) * h_{b 1}(t) \\
x_{w}(t) * h_{12}(t)+x_{b}(t) * h_{b 2}(t)
\end{array}\right] } \\
(2) & =\left[\begin{array}{l}
x_{w}(t) * h_{11}(t)+(m(t) \cdot z(t)) * h_{b 1}(t) \\
x_{w}(t) * h_{12}(t)+(m(t) \cdot z(t)) * h_{b 2}(t)
\end{array}\right] \\
\text { (3) }= & {\left[\begin{array}{l}
x_{w}(t) * h_{11}(t)+\left(m(t) \cdot\left(x_{w}(t) * h_{f}(t)\right)\right) * h_{b 1}(t) \\
x_{w}(t) * h_{12}(t)+\left(m(t) \cdot\left(x_{w}(t) * h_{f}(t)\right)\right) * h_{b 2}(t)
\end{array}\right] } \\
\text { (4) }= & {\left[\begin{array}{l}
x_{w}(t) * h_{11}(t)+m(t) \cdot\left(x_{w}(t) *\left[h_{f}(t) * h_{b 1}(t)\right]\right) \\
x_{w}(t) * h_{12}(t)+m(t) \cdot\left(x_{w}(t) *\left[h_{f}(t) * h_{b 2}(t)\right]\right)
\end{array}\right] . }
\end{aligned}
$$




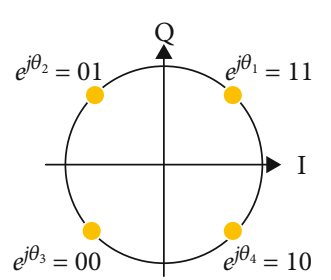

FIGURE 4: Constellation diagram of 4-PSK and bits represented by constellation points.

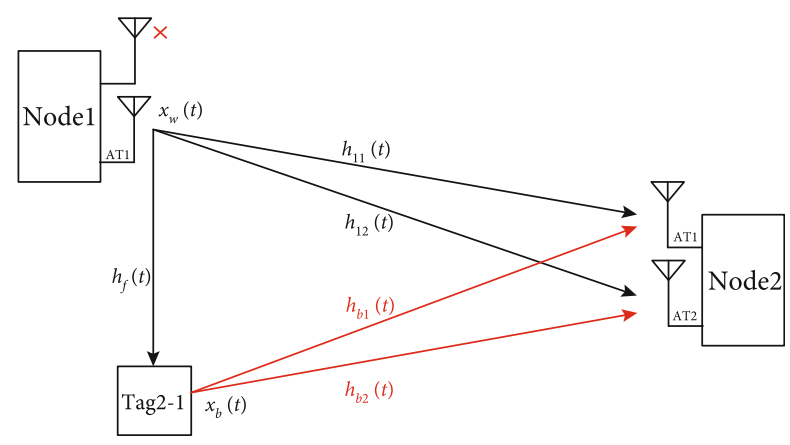

FIgURE 5: A MU-MIMO system in the tag-to-WiFi communication: Node1 and Tag2-1 are inputs, and Node2's AT1 and AT2 are outputs.

In (6), $h_{1 i}(t)$ is the impulse response of the channel between Nodel's AT1 and Node2's ATi, $i=1,2$, and $h_{b i}(t)$ is the impulse response of the channel between the tag and Node2's ATi, $i=1,2$. According to (1), we transform (1) to (2). Then, we have (3) from (2), because $z(t)$ is $x_{w}(t)$ received at the tag, where $z(t)=x_{w}(t) * h_{f}(t)$ and $h_{f}(t)$ is impulse response of the channel between Nodel's AT1 and the tag. Next, because the symbol period is much longer than the typical propagation delay in IoT (e.g., $50 \mathrm{~ns}$ in $\mathrm{WiFi}[8]$ ), we can consider $m(t)$ to be a constant [8] and hence have (3) to (4). Finally, if $h_{1 i}(t)$ and $h_{f}(t) * h_{b 1}(t), i=1,2$ in equation (4) are known, we can obtain $x_{w}(t)$ and $m(t)$ by combining these equations. Fortunately, benefitting from MAC layer design (see Section 2.3), $h_{1 i}(t)$ and $h_{f}(t) * h_{b 1}(t), i=1,2$, can be estimated before demodulations.

At the receiver end, analog signals are sampled before being further processed. Hence, we can write (6) in the discrete domain as follows:

$$
\mathbf{y}=\left[\begin{array}{l}
x_{w}[n] \cdot h_{11}+m[n] \cdot\left(x_{w}[n] \cdot\left[h_{f} \cdot h_{b 1}\right]\right) \\
x_{w}[n] \cdot h_{12}+m[n] \cdot\left(x_{w}[n] \cdot\left[h_{f} \cdot h_{b 2}\right]\right)
\end{array}\right] .
$$

Step 2. Decoding backscatter data.

Upon extracting $m[n]$, we perform the following operations to decode bits from it. First, we, respectively, compute the distance between $m[n]$ and each of valid constellation points in e. Then, we regard $m[n]$ as the constellation point that results in the shortest distance and decode $m[n]$ as the corresponding bits. For example, if $m[n]$ is closest to $e^{j \theta_{4}} \in \mathbf{e}$ and " 10 " corresponds to $e^{j \theta_{4}}$, we decode $m[n]$ as " 10 ."
2.3. MAC Layer Design. To better describe our MAC layer design, we assume an IoT network with the topology shown in Figure 2. In this topology, the IoT network comprises two subcells, i.e., subcell $k, k=1,2$. In subcell $k$, there are one WiFi node (i.e., Node $k$ ) and two tags (i.e., Tag $k-1$ and Tag $k-2)$.

Further, we describe the MAC layer design using the example shown in Figure 6. In this example, Node1 and Node2 perform carrier sense multiple access/collision avoidance (CSMA/CA) mechanism to contend for their transmissions. Then, Nodel wins the contention and the whole network starts a process that can be divided into four periods as follows.

2.3.1. Period (1): Header Transmission. In this period, Node1 sends a WiFi PHY header (including WiFi preamble) and a MAC header. All tags (i.e., Tag1-1, Tag1-2, Tag2-1, and Tag2-2) harvest energy from the WiFi signal carrying headers. Node2 receives the $\mathrm{PHY}$ header and uses the WiFi preamble in the header to evaluate $h_{1 i}(t), i=1,2$, in Equation (6).

2.3.2. Period (2): Asynchronous Block $A C K$ (AB-ACK). In this period, Node1 sends an asynchronous block ACK (AB-ACK) shown in Figure 7, which comprises a Start field (i.e., the start of the tag and WiFi transmission), a Transmit address field (TA for short, i.e., the signature of Node1), a Duration field (i.e., the number of Receive address), and $n$ Receive address fields (RA for short, i.e., the signature of the sender of the backscattered data buffered in Node1). The length of each field is 1 microslot. In the example of Figure 6, the RA only contains the signature of Tag1-1 (i.e., $n=1$ ), indicating that Node1 has only successfully received and buffered the backscatter data from Tag1-1 between its last and current transmission.

All tags receive the $\mathrm{AB}-\mathrm{ACK}$ and detect the TA field in it. Tag2-1 and Tag2-2 learn from TA that the current sender node does not belong to the same subcell (i.e., is not Node2), so they read $n$ from the duration field and then perform energy harvesting operation for a time of sending $n$ RAs (i.e., $n$ microslots). Meanwhile, Tag1-1 and Tag1-2 learn from TA that Node1 is the current sender node, so they continue to check the Duration and RA fields. Then, Tag1-1 detects its signature in RA and knows that its previous transmission is successful. Meanwhile, Tag1-2 does not detect its signature in RA and hence knows that its previous transmission is unsuccessful. Later, it will perform a retransmission to Nodel.

Node2 keeps silent.

2.3.3. Period (3): Backscatter Contention. This period is further divided into $l$ microslots. In each microslot, Node1 sends a predefined busy tone.

Tag1-1 and Tag1-2 perform energy harvesting and do not send data to Node1, because Node1 is performing transmission instead of reception. Meanwhile, Tag2-1 and Tag2-2 attempt to send packet to Node2; hence, they perform a contention as follows. First, they generate a random backoff counter uniformly distributed in $(0, l-1)$. Then, they sense the channel and decrease their counters by 1 if there is no backscatter preamble (B-preamble) being transmitted. The B-preamble is a predefined special sequence transmitted 


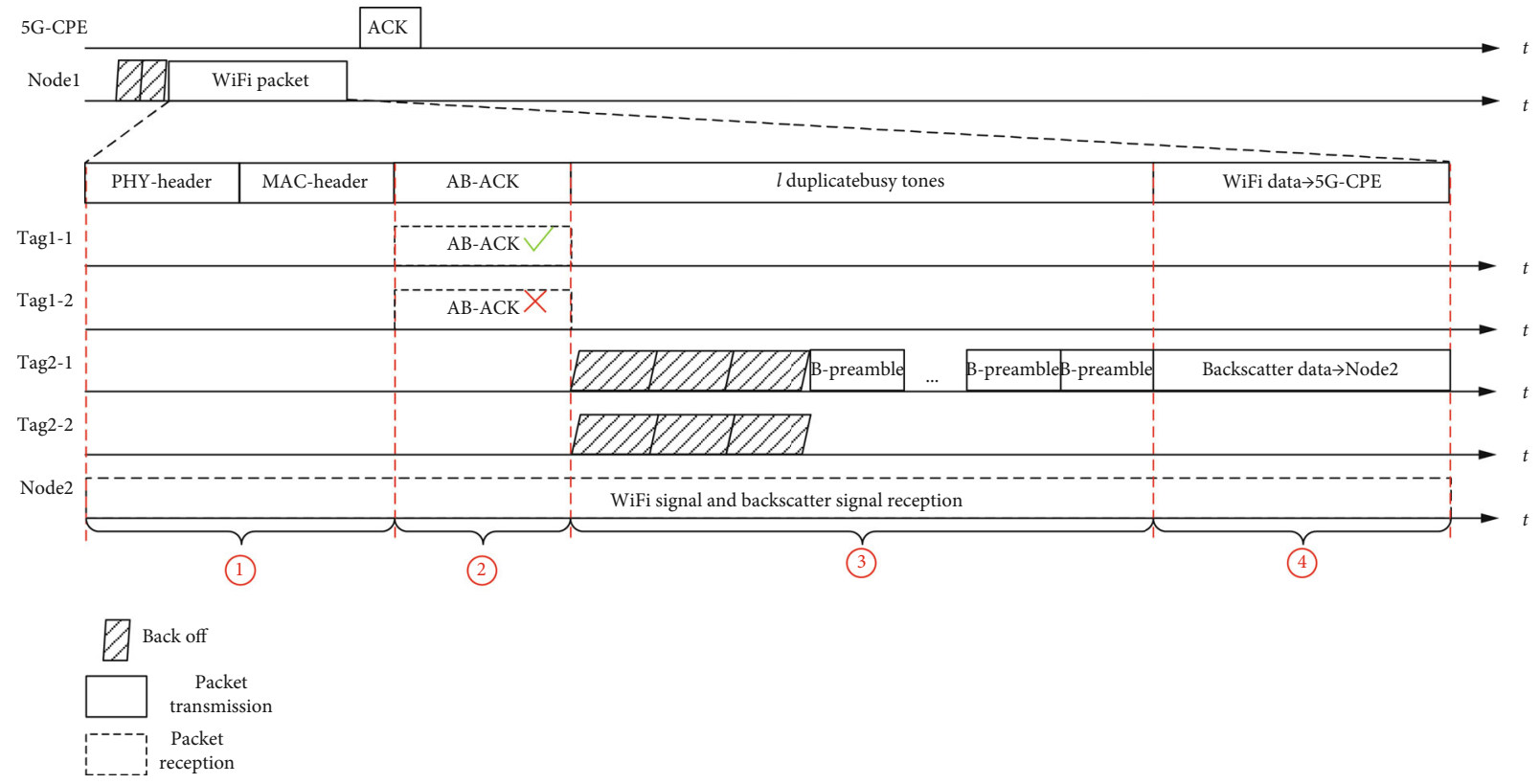

FIGURE 6: Example of transmission process in our MAC design.

\begin{tabular}{|c|c|c|c|c|c|}
\hline 1 & \multicolumn{3}{c|}{} \\
\hline Start & Transmit address (TA) & Duration & Receive address 1 (RA1) & $\ldots$ & Receive address $n($ RA $n)$ \\
\hline
\end{tabular}

FIgURE 7: Asynchronous block ACK format.

using our tag-to-tag communication scheme and its length is 1 microslot. Then, Tag2-1's counter first reaches 0 and Tag21 starts to broadcast B-preamble repeatedly until the $l$ th microslot. Tag2-2 detects Tag2-1's B-preambles, stops its backoff and transmission attempt, and performs energy harvesting.

Node2 receives a busy tone from Node1 and a Bpreamble from Tag2-1 in the $l$ th microslot. Then, it substitutes them and the $h_{1 i}(t), i=1,2$ obtained in period (1) into (4) to estimate $h_{f}(t) * h_{b i}(t), i=1,2$.

2.3.4. Period (4): Backscatter Transmission. In this period, Node1 follows the WiFi standard to send its data and the buffered Tag1-1's data to 5G-CPE. Tag1-1, Tag1-2, and Tag2-2 perform energy harvesting. Tag2-1 sends a packet to Node2 using our tag-to-WiFi communication scheme, where the packet consists of the tag's address and a data payload. Node 2 buffers this packet and records the tag's address, and then, it forwards the packet to the 5G-CPE in its next transmission.

\section{Performance Analysis}

In this section, we analyze the MAC layer performance of the proposed backscatter system (namely, throughput). We consider a saturated network (i.e., all WiFi nodes and backscatter tags always have a packet to send) consisting of $15 \mathrm{G}-\mathrm{CPE}$ and $n$ subcells. Each subcell consists of 1 node and $g$ tags. That is, there are $n$ nodes and $G$ tags in total, where $G=n$ $\times g$. We also assume that the nodes have the same packet size $L_{\mathrm{w}}$ and the tags have another same packet size $L_{\mathrm{b}}$. In the following, we express the throughput in terms of WiFi and backscatter.

3.1. Throughput of WiFi. In the following, we first calculate the generic slot and then express the throughput of $\mathrm{WiFi}$.

3.1.1. Calculation of the Generic Slot. Let $\tau(\gamma)$ denote the attempt rate of each WiFi node. In 802.11 networks, $\tau(\gamma)$ can be achieved through setting the minimum contention windows $\left(\mathrm{CW}_{\text {min }}\right)$ and the maximum backoff stage $(m)$ [21]. We have

$$
\tau(\gamma)=\frac{2}{1+\mathrm{CW}_{\min }+\gamma \mathrm{CW}_{\min } \sum_{k=0}^{m-1}(2 \gamma)^{k}},
$$

where $\gamma$ is the collision probability of each WiFi node and can be expressed as

$$
\gamma=1-(1-\tau(\gamma))^{n-1}
$$

Let $\Omega$ denote time duration of the generic slot that elapses for one decrement of the backoff counter. $\Omega$ depends on 
TABLE 1: Main simulation parameters for PHY and MAC layers of WiFi.

\begin{tabular}{lcc}
\hline Parameter & Description & Value \\
\hline PHY model & Data rate of MCS $4 / \mathrm{MCS} 8$ & $3.9 / 7.8 \mathrm{Mb} / \mathrm{s}$ \\
$R_{\text {data }}$ & Basic rate & $0.65 \mathrm{Mb} / \mathrm{s}$ \\
$R_{\text {basic }}$ & Length of PHY header & $160 \mu \mathrm{s}$ \\
PHY header & & \\
MAC model & Duration of SIFS interval \\
$T_{\text {SIFS }}$ & Duration of DIFS interval \\
$T_{\text {DIFS }}$ & Duration of an IEEE 802.11 ah time slot \\
$T_{\text {Slot }}$ & Length of DATA packets \\
$L_{\text {WiFi }}$ & Length of ACK packets \\
$L_{\text {ACK }}$ & $(40$ bytes +4 bytes $) @ R_{\text {data }}$ \\
MAC header & 20 bytes@ $R_{\text {data }}$ \\
Route header & 1000 bytes@ $R_{\text {data }}$ \\
$T_{\text {WiFi }}$ & $\left(24\right.$ bytes +14 bytes $@ @ R_{\text {basic }}$ \\
$T_{\text {ACK }}$ & Minimum value of contention window \\
CW & Maximum backoff stage \\
$m$ & & 1000 bytes \\
\hline
\end{tabular}

whether a slot is idle or interrupted by a successful or an unsuccessful WiFi transmission [22, 23]. So, $\Omega$ can be expressed as

$$
\Omega=\left\{\begin{array}{l}
\sigma, 1-P_{\mathrm{b}}, \\
T_{\mathrm{s}}, P_{\mathrm{s}}, \\
T_{\overline{\mathrm{s}}}, P_{\mathrm{b}}-P_{\mathrm{s}},
\end{array}\right.
$$

where $P_{\mathrm{b}}=1-(1-\tau(\gamma))^{n}$ is the probability of a busy slot; $P_{\mathrm{s}}=n \tau(\gamma)(1-\tau(\gamma))^{n-1}$ is the probability of a successful WiFi transmission from any of $n$ contending nodes; $\sigma$ is the duration of one time slot; and $T_{\mathrm{s}}$ and $T_{\overline{\mathrm{s}}}$ are the mean time for a successful transmission and an unsuccessful transmission, respectively.

Let $E(\Omega)$ denote the mean value of $\Omega$. We have

$$
E(\Omega)=\left(1-P_{\mathrm{b}}\right) \sigma+P_{\mathrm{s}} T_{\mathrm{s}}+\left(P_{\mathrm{b}}-P_{\mathrm{s}}\right) T_{\overline{\mathrm{s}}} .
$$

3.1.2. Calculation of WiFi's Throughput. We define the throughput, $\Gamma_{\mathrm{w}}$, as the number of bits that the WiFi nodes successfully transmit in $E(\Omega)$ [21-23]. We have

$$
\Gamma_{\mathrm{w}}=\frac{P_{\mathrm{s}} L_{\mathrm{w}}}{E(\Omega)} .
$$

3.2. Throughput of Backscatter. Here, we first calculate the probability of a successful backscatter transmission and then calculate the throughput of backscatter.

3.2.1. Calculation of the Probability of a Successful Backscatter Transmission. In a subcell, when there is only one tag that wins the contention, the winner tag performs a successful backscatter transmission. Let $\beta$ denote the probability of a successful backscatter transmission. We have

$$
\beta=\frac{\sum_{i=0}^{l-2} C_{g}^{1}\left(C_{l-i-1}^{1}\right)^{g-1}}{\left(C_{l}^{1}\right)^{g}} .
$$

In (13), the denominator represents the number of $g$ tags' all possible contention results, while the numerator represents the number of that only one tag wins the contention. In the numerator, $i$ is the backoff counter of the winner; $l-$ 2 is the maximum backoff counter that the winner can select. $C_{g}^{1}$ represents that any one of $g$ contending tags wins the contention, where its backoff counter equals to $i$. $\left(C_{l-i-2}^{1}\right)^{g-1}$ represents that the other $g-1$ tags fail the contention, where their backoff counters are greater than $i$.

3.2.2. Calculation of Backscatter's Throughput. Each successful WiFi transmission can provide a backscatter Transmission Opportunity (TXOP) for all winner tags in other $n-1$ subcells (except the subcell of the WiFi transmitter), and then, the winners can transmit their backscatter data to the neighbouring WiFi nodes in parallel. Note that we have the same $\beta$ in all subcells, because the number of tags in all subcells is the same. Therefore, we express the throughput of backscatter, $\Gamma_{\mathrm{b}}$, by

$$
\Gamma_{\mathrm{b}}=\frac{(n-1) \beta P_{\mathrm{s}} L_{\mathrm{b}}}{E(\Omega)} .
$$

Finally, we also define the total throughput, $\Gamma_{\mathrm{t}}$, as the sum of WiFi's throughput and backscatter's throughput, which can be expressed as 


$$
\Gamma_{\mathrm{t}}=\Gamma_{\mathrm{w}}+\Gamma_{\mathrm{b}}
$$

\section{Performance Evaluation}

In this section, we verify the accuracy of our theoretical model and make comparisons with BackFi [8].

In our design, we consider an IoT network consisting of 1 5G-CPE, $n$ nodes, and $G(G=n \times g)$ tags, where each subcell consists of 1 node and $g$ tags. The WiFi nodes contend to upload their data to 5G-CPE, where each WiFi transmission activates $n-1$ backscatter transmissions in other subcells.

In BackFi, the IoT network comprises 1 5G-CPE, $n$ nodes, and $g$ tags, where all tags surround the 5G-CPE in the transmission range of backscatter. The $5 \mathrm{G}-\mathrm{CPE}$ of BackFi contends for the channel to obtain the backscatter data. When the 5G-CPE wins the channel, it sends the excitation signal to a target tag. Then, the tag modulates the excitation signal and reflects the signal back to the $5 \mathrm{G}-\mathrm{CPE}$.

In simulation, we consider a saturated network (in both our design and BackFi). The PHY and MAC layer's default parameter settings of our design and BackFi are set according to IEEE 802.11ah [13] and listed in Table 1. The other default parameter settings of our design are shown in Table 2.

Here, we verify the accuracy of our model in terms of throughputs (which are calculated by (12) and (14)). Figure 8 plots the WiFi's throughput and backscatter's throughput, when $n=1, \cdots, 10 ; g=5$; and $R_{\text {data }}=3.9 \mathrm{Mb} / \mathrm{s}$. From Figure 8, we have the following observations:

(i) The theoretical curves closely match the simulation curves. This manifests that our performance model is very accurate

(ii) As $n$ increases, the throughput of $\mathrm{WiFi}$ decreases slightly, while the throughput of backscatter increases significantly. For example, the throughput of backscatter when $n=10(0.68 \mathrm{Mb} / \mathrm{s})$ is 0.7 times more than that of when $n=5(0.4 \mathrm{Mb} / \mathrm{s})$

Here, we compare our design with BackFi in terms of WiFi's throughput, backscatter's throughput, and total throughput. Figure 9 plots the throughput of our design and BackFi as the number of nodes, $n$, varies, where $g=5$ and $R_{\text {data }}=7.8 \mathrm{Mb} / \mathrm{s}$. From Figure 9, we have the following observations:

(i) As $n$ increases from 1 to 20, the total throughput of our design is steady at $3.0 \mathrm{Mb} / \mathrm{s}$, while that of BackFi decreases from $3.1 \mathrm{Mb} / \mathrm{s}$ to $2.5 \mathrm{Mb} / \mathrm{s}$

(ii) The backscatter's throughput of our design is much higher than that of BackFi. The reasons are as follows. In our design, each $\mathrm{WiFi}$ transmission (whether transmitted by the 5G-CPE or the WiFi nodes) can activate $n-1$ backscatter transmissions. However, in BackFi, only each 5G-CPE's transmission can activate one backscatter transmission

(iii) The WiFi's throughput of our design is always $0.3 \mathrm{Mb} / \mathrm{s}$ lower than that of BackFi. This is because
TABLE 2: Main simulation parameters of our design.

\begin{tabular}{lcc}
\hline Parameter & Description & Value \\
\hline$l$ & Number of microslot & 8 \\
$L_{\text {micro-slot }}$ & Length of micro-slot & $16 \mu \mathrm{s}$ \\
\hline
\end{tabular}

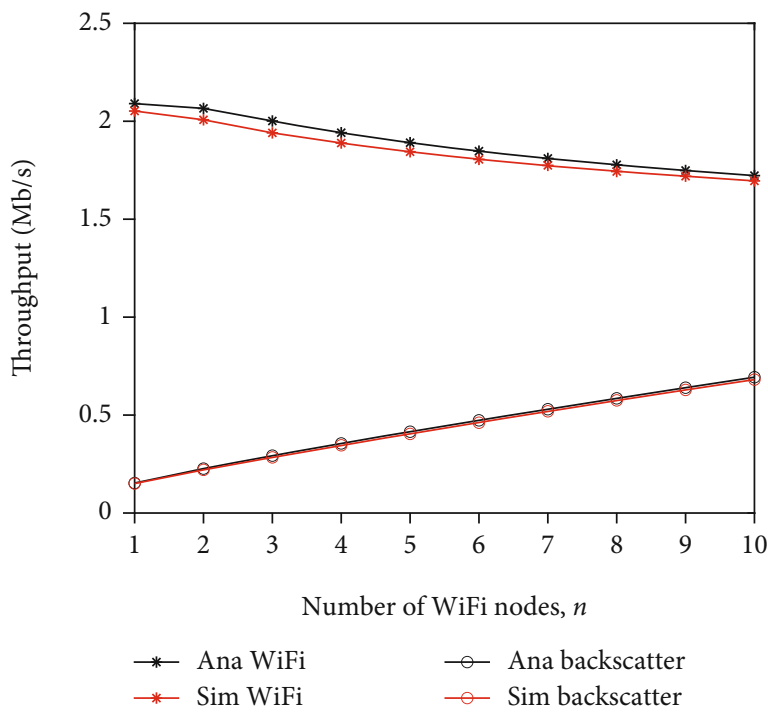

Figure 8: Throughput vs. number of WiFi nodes.

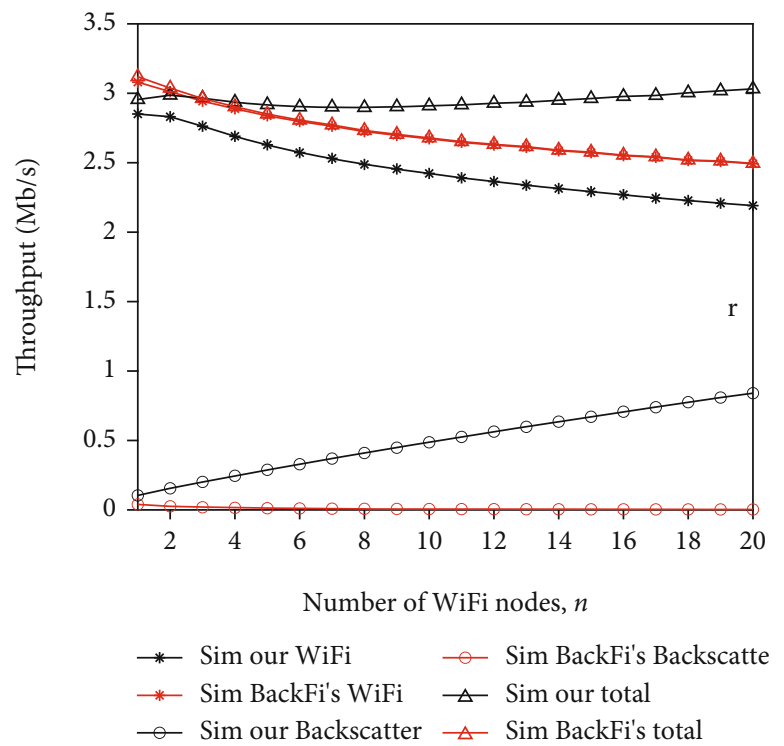

Figure 9: Comparison of throughput between our design and BackFi.

our design introduces the AB-ACK and the backscatter contention, which increases redundancy of WiFi packet

\section{Conclusions}

In this paper, we propose a low-cost and long-range nodeassisted WiFi-BSC for 5G-enabled IoT. First, in the PHY 
layer, we enable the off-the-shelf WiFi devices to receive and decode backscatter signals using only two regular antennas, which reduces the deployment cost of WiFi-BSC significantly. Then, in the MAC layer, we enable WiFi nodes to relay WiFi-BSC data toward the 5G-CPE, extending the communication range of WiFi-BSC greatly. Next, we model our scheme. Finally, we run extensive simulations to verify the accuracy of our model and the effectiveness of our scheme. This study shows that the WiFi-BSC has a great potential in 5G-enabled IoT.

\section{Data Availability}

The data used to support the findings of this study are available from the corresponding author upon request.

\section{Conflicts of Interest}

The authors declare that there are no conflicts of interest regarding the publication of this paper.

\section{Acknowledgments}

This work is funded in part by the National Nature Science Foundation of China (File no. 61872451 and 61872452) and in part by the Science and Technology Development Fund, Macau SAR (File nos. 0098/2018/A3, 0037/2020/A1, and 0062/2020/A2).

\section{References}

[1] J. Ni, X. Lin, and X. S. Shen, "Efficient and secure serviceoriented authentication supporting network slicing for 5Genabled IoT," IEEE Journal on Selected Areas in Communications, vol. 36, no. 3, pp. 644-657, 2018.

[2] "Minimum requirements related to technical performance for IMT-2020 radio interface(s)," https://www.itu.int:443/en/ publications/ITU-R/Pages/publications.aspx.

[3] "5G for connected industries and automation white paper," https://www.5g-acia.org/fileadmin/5G-ACIA/Publikationen/ Whitepaper_5G_for_Connected_Industries_and_ Automation/WP_5G_for_Connected_Industries_and_ Automation_Download_19.03.19.pdf.

[4] M. Simsek, A. Aijaz, M. Dohler, J. Sachs, and G. Fettweis, “5Genabled tactile internet," IEEE Journal on Selected Areas in Communications, vol. 34, no. 3, pp. 460-473, 2016.

[5] D. B. Lukic, G. B. Markovic, and D. D. Drajic, "Two-stage precoding based on overlapping user grouping approach in IoToriented 5G MU-MIMO systems," Wireless Communications and Mobile Computing, vol. 2021, Article ID 8887445, 13 pages, 2021.

[6] J. Yang, L. Feng, T. Jin, H. Liang, F. Xu, and L. Tian, "Enabling sector scheduling for 5G-CPE dense networks," Security and Communication Networks, vol. 2020, Article ID 6684681, 18 pages, 2020.

[7] V. Liu, A. Parks, V. Talla, S. Gollakota, D. Wetherall, and J. R. Smith, "Ambient backscatter: wireless communication out of thin air," ACM SIGCOMM Computer Communication Review, vol. 43, no. 4, pp. 39-50, 2013.
[8] D. Bharadia, K. R. Joshi, M. Kotaru, and S. Katti, "BackFi: high throughput WiFi backscatter," ACM SIGCOMM Computer Communication Review, vol. 45, no. 4, pp. 283-296, 2015.

[9] P. Zhang, D. Bharadia, K. Joshi, and S. Katti, "HitchHike: practical backscatter using commodity WiFi," in Proceedings of the 14th ACM Conference on Embedded Network Sensor Systems CD-ROM, pp. 259-271, Stanford, CA, USA, 2016.

[10] B. Kellogg, A. Parks, S. Gollakota, J. R. Smith, and D. Wetherall, "Wi-Fi backscatter: internet connectivity for RF-powered devices," ACM SIGCOMM Computer Communication Review, vol. 44, pp. 607-618, 2014.

[11] Z. Ma, L. Feng, and F. Xu, "Design and analysis of a distributed and demand-based backscatter MAC protocol for internet of things networks," IEEE Internet of Things Journal, vol. 6, no. 1, pp. 1246-1256, 2018.

[12] E. Khorov, A. Lyakhov, A. Krotov, and A. Guschin, "A survey on IEEE 802.11ah: an enabling networking technology for smart cities," Computer Communications, vol. 58, pp. 53-69, 2015.

[13] T. Adame, A. Bel, B. Bellalta, J. Barcelo, and M. Oliver, "IEEE 802.11AH: the WiFi approach for M2M communications," IEEE Wireless Communications, vol. 21, no. 6, pp. 144-152, 2014.

[14] N. van Huynh, D. T. Hoang, X. Lu, D. Niyato, P. Wang, and D. I. Kim, "Ambient backscatter communications: a contemporary survey," IEEE Communications Surveys \& Tutorials, vol. 20, no. 4, pp. 2889-2922, 2018.

[15] X. Li, M. Zhao, Y. Liu, L. Li, Z. Ding, and A. Nallanathan, "Secrecy analysis of ambient backscatter NOMA systems under I/Q imbalance," IEEE Transactions on Vehicular Technology, vol. 69, no. 10, pp. 12286-12290, 2020.

[16] X. Li, M. Zhao, M. Zeng et al., "Hardware impaired ambient backscatter NOMA systems: reliability and security," IEEE Transactions on Communications, vol. 69, no. 4, pp. 2723 2736, 2021.

[17] X. Li, Y. Zheng, W. U. Khan et al., "Physical layer security of cognitive ambient backscatter communications for green Internet-of-Things," IEEE Transactions on Green Communications and Networking, 2021.

[18] X. Cao, Z. Song, B. Yang, M. A. Elmossallamy, L. Qian, and Z. Han, "A distributed ambient backscatter MAC protocol for Internet-of-Things networks," IEEE Internet of Things Journal, vol. 7, no. 2, pp. 1488-1501, 2019.

[19] X. Cao, Z. Song, B. Yang, M. ElMossallamy, L. Qian, and Z. Han, "A distributed MAC using Wi-Fi to assist sporadic backscatter communications," in IEEE INFOCOM 2019-IEEE Conference on Computer Communications Workshops (INFOCOM WKSHPS), pp. 780-785, Paris, France, 2019.

[20] X. Cao, Z. Song, B. Yang, X. Du, L. Qian, and Z. Han, “Deep reinforcement learning mac for backscatter communications relying on Wi-Fi architecture," in 2019 IEEE Global Communications Conference (GLOBECOM), pp. 1-6, Waikoloa, HI, USA, 2019.

[21] G. Bianchi, "Performance analysis of the IEEE 802.11 distributed coordination function," IEEE Journal on Selected Areas in Communications, vol. 18, no. 3, pp. 535-547, 2000.

[22] Q. Zhao, D. H. Tsang, and T. Sakurai, "A simple and approximate model for nonsaturated IEEE 802.11 DCF," IEEE Transactions on Mobile Computing, vol. 8, no. 11, pp. 1539-1553, 2009.

[23] Q. Zhao, D. H. Tsang, and T. Sakurai, "Modeling nonsaturated IEEE 802.11 DCF networks utilizing an arbitrary buffer size," IEEE Transactions on Mobile Computing, vol. 10, no. 9, pp. 1248-1263, 2010. 